\title{
RIGHTING OF CHINESE MITTEN CRABS (ERIOCHEIR SINENSIS) AND THEIR MODELS
}

\author{
Tobias Riphaus ${ }^{a, *}$, Florian Hoffmann ${ }^{b}$, Susanna Labisch ${ }^{b}$ \\ ${ }^{a}$ International Degree Course in Biomimetics, City University of Applied Science Bremen, Germany \\ ${ }^{b}$ Biomimetics-Innovation-Centre (B-I-C), City University of Applied Science Bremen, Germany \\ * corresponding author: T. Riphaus via slabisch@bionik.hs-bremen.de
}

\begin{abstract}
The usage of unmanned underwater vehicles for marine tasks is continuously growing and bioinspired stabilizing systems shall help them to gain and keep a stable position during work. Therefore the righting maneuver of E. sinensis has been studied. These crabs are able to perform a $180^{\circ}$-rotation with an angular velocity of $4.30 \mathrm{~s}^{-1}$ when falling underwater from a supine starting position. High-speed particle image velocimetry has shown, that propulsive forces with a peak of 0.021 $\pm 0.001 \mathrm{~N}$ were produced by the hind legs to initiate and stop the rotation. In a numerical multibody simulation a constant force of $0.009 \mathrm{~N}$ acting for $0.2 \mathrm{~s}$ leads to the same rotation. In order to prove this mechanism, it was implemented into a robotic system. Its mean density of $1.15 \mathrm{~g} / \mathrm{cm}^{3}$ deviates not more than $4 \%$ from the biological and numerical models. It can complete a $180^{\circ}$-turn within $1.03 \pm$ $0.12 \mathrm{~s}$ with a rotational velocity of up to $4.25 \mathrm{~s}^{-1}$.
\end{abstract}

KEYWORDS: biomimetics, righting maneuver, robot stabilization.

\section{INTRODUCTION}

Since the first success of unmanned underwater vehicles (UUVs) their field of operation is constantly spreading. In spite of all the technology, the optimization of their stabilizing behavior is still a crucial topic in contemporary research. The reason why even modern UUVs are facing problems in holding stable positions, to carry out their tasks or turn around quickly once they have lost their preferred position, is the kind of drive that is mainly used. Propeller and pump-jets are advantageous for high-speed locomotion, but too expensive for the use in a seldom occurring event.

To gain new ideas for solving such problems more and more research groups are investigating biological models. First successful terrestrial applications are the amphibious robot RoboCrab by Krummel et al., which adapts the skills of horseshoe crabs within the surf zone, or the four-wheeled autonomous robot by ChangSiu et al., which is capable of mid-air reorienting during free fall modeled on geckoes (Hemidactylus platyurus) [1] [2].

But the usage of biological models for improving aquatic robots is poorly documented. Thus, the research group DFU of the Department of Biomimetics in Bremen started investigations on different benthic marine animals. The aim was to search for a strategy that allows a quick turning or efficient reorientation manoeuvre which uses neither a propeller nor is driven by a permanent rotation. Especially promising seemed the crustacean order Decapoda. Therefore the species Eriocheir sinensis has been studied in terms of their mid-water righting behavior [3] [4]. Besides the morphological biomechanical analysis, also the necessary

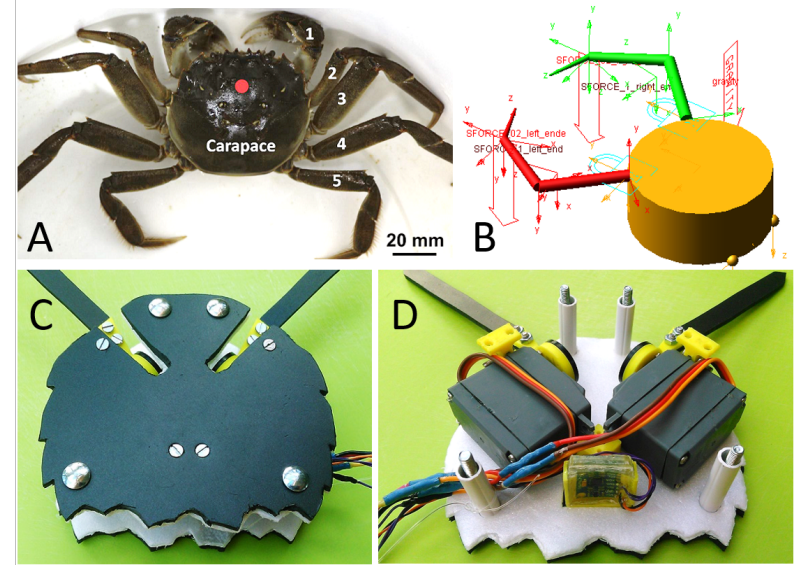

Figure 1. Individual of E. sinensis (A, 3 modified), its numerical model (B) 4 and the adapted robot O-Typus $(\mathbf{C})$ with an insight into its electronic devices $(\mathbf{D})[5]$.

hydrodynamic forces have to be determined to show, that such a strategy is transferable to a technical application.

\section{MAterial AND Methods}

\subsection{Eriocheir sinensis - BIOLOGICAL MODEL}

In total 52 rotations of 10 individuals of E. sinensis were analysed. The rotations are differentiated in the kinematic approach with 39 analysed rotations (28/11, initialising/stopping rotation) and for the PIV (Particle Image Velocimetry) analysis with 13 analysed rotations $(9 / 4$, initialising/stopping rotation). 


\subsubsection{KINEMATIC ANALYSIS}

To observe the natural righting behavior of E. sinensis (Figure 1A), the crabs were held in a supine starting position at the height of $0.60 \mathrm{~m}$ within a freshwater tank with a ground area of $0.6 \times 0.6 \mathrm{~m}$ and a high of $0.8 \mathrm{~m}$ and released by starting device. The body movement was high speed recorded (250 fps) to be able to manually track two points on the body and the tip of the 5th leg. Furthermore falling speed, body angle and leg trajectories were plotted and the speed of the leg tip calculated. The leg induced force $\left(\mathrm{F}_{d}\right)$ during the strike was calculated by

$$
F_{d}=\frac{1}{2} \rho v_{c}^{2} c_{d} A
$$

where $\rho=$ water density, $v_{c}=\operatorname{leg}$ centroid velocity, $\mathrm{A}=$ area of 5 th legs and $\mathrm{c}_{d}=$ drag coefficient from static force balance measurements.

Centroid velocity was calculated by:

$$
v_{c}=v_{t} \frac{L_{b c}}{L_{b t}}
$$

where $v_{c}=$ velocity of centroid, $v_{t}=$ velocity of leg tip, $\mathrm{L}_{b c}=$ length leg base to leg centroid and $\mathrm{L}_{b t}=$ length leg base to leg tip.

$\mathrm{L}_{b t}$ and $\mathrm{L}_{b c}$ were experimental determined on 15 legs of 10 dead E. sinensis. Centroid x/y coordinates were calculated with an integrated function of ImageJ from the leg area. The resulting factor for $\mathrm{L}_{b c} / \mathrm{L}_{b t}$ was calculated as $0.44 \pm 0.016$.

\subsubsection{PiV MEASUREMEnts AND FOrCE CALCULATION}

The tank of the kinematic study was also used for the PIV measurements. Crabs were released by hand or the automated release fixture. The laser sheet was placed vertically in the middle of the tank illuminating a surface of approx. $0.3 \times 0.3 \mathrm{~m}$. Flow was visualized by Polyamid Seeding particles ( $\varnothing 57 \mu \mathrm{m}$, density: $1.03 \mathrm{~g} / \mathrm{cm}^{3}$ ). Images were taken by a high speed camera (Photron APX-RS) at an interframing time of $3.75 \mathrm{~ms}$ (266 fps). Crabs were released at different distances to the lasersheet depending on the leg length. We used falling sequences where the last leg was cutting the lasersheet at positions from 50 to $80 \%$ of the leg. By measuring the bound circulation forces around the leg the generated propulsion force was calculated by using the Kutta-Joukowski theorem. Circulation $\Gamma$ was measured from the beginning to the end of the leg stroke in order to get a temporal resolution of the circulation.

In PIVLab [6] under MATLAB (The MathWorks Inc.) the PIV images were analysed and the spanwise circulation (circulation around the spanwise axis) was derived by a loop integral of the tangent velocity:

$$
\Gamma=\oint_{S_{\text {vort }}} v_{t} d S_{\text {vort }}=\int_{A_{\text {vort }}} \omega_{Z} d A_{\text {vort }}
$$

where $S_{\text {vort }}=$ circular path around the vortex core, $v_{t}=$ tangential velocity, $A_{\text {vort }}=$ area of the vortex core and $\omega_{Z}=$ spanwise vorticity.

The vortex ring momentum calculation model was used to estimate an average propulsive force [7] [8]. The average propulsive force of the leg stroke was herewith calculated by

$$
F=2 \Gamma \rho \nu b
$$

where $\Gamma=$ circulation, $\rho=$ fluid density, $\nu=$ fluid velocity and $b=$ leg length. The force results were multiplied by two, to get the force of both legs.

\subsection{Simulated Model}

For the numerical simulation of the physical behavior the software ADAMS (rel. 2013.1) was used. It allows to describe the limb movement as a motion of mechanically joint rigid bodies, builds the relevant differential equations of motion and solves them numerically.

Basis for the multi-body-analysis is experimental gained data of an individual crab showing the dimensions, the mass, the density and the center of mass (COM) of each limb. All body-parts are connected with rigid joints. In order to simulate the correct falling speed in water the gravity was reduced to 1.09 $\mathrm{m} / \mathrm{s}^{2}$.

During movement the crabs hold their front legs close to the carapax and stretching out only the hind legs. Therefore the main body can easily be simplified to a cylinder. With a diameter of $6.00 \mathrm{~cm}$ and a height of $2.75 \mathrm{~cm}$ for carapax and front legs such a cylinder will have an average density of $1.12 \mathrm{~g} / \mathrm{cm}^{3}$ which leads to a mass of $87 \mathrm{~g}$. This data fits the measured crab very well. The hind leg pair is modelled with the merus/ischium, the propodus/carpus and the dactylus in their measured length and density. The parts of the leg are conical so that the COM of these parts matches also (Figure 1B). The acting forces are applied on the joint between dactylus and propodus with a constant value for a given duration (thick arrows in Fig $1 \mathrm{~B}$ ).

\subsection{O-Typus - Technical Model}

\subsubsection{SETUP}

To confirm whether the observed mechanism is correctly understood, the basic model is implemented in the purpose-built robot O-Typus (Figure $1 \mathrm{C}+\mathrm{D}$ ) [5]. Its chassis is congruent with the carapace of an individual of E. sinensis and scaled 2:1, to house drive units and the sensor system.

The actuating elements consist of two waterproof HK15328D servo motors (max torque: $12,8 \mathrm{~kg} . \mathrm{cm}$ ) with a $6 \mathrm{~V}$ external power supply $(2250 \mathrm{~mA})$. Their control is based on the PWM-signals from an Arduino Uno. The position tracking is enabled by the MPU6050 accelerometer. The real time operation sends the current Yaw-/ Pitch-/ Roll-Angles (YPR) to the Arduino Uno to log the data $(\sim 27 \mathrm{~Hz})$. 

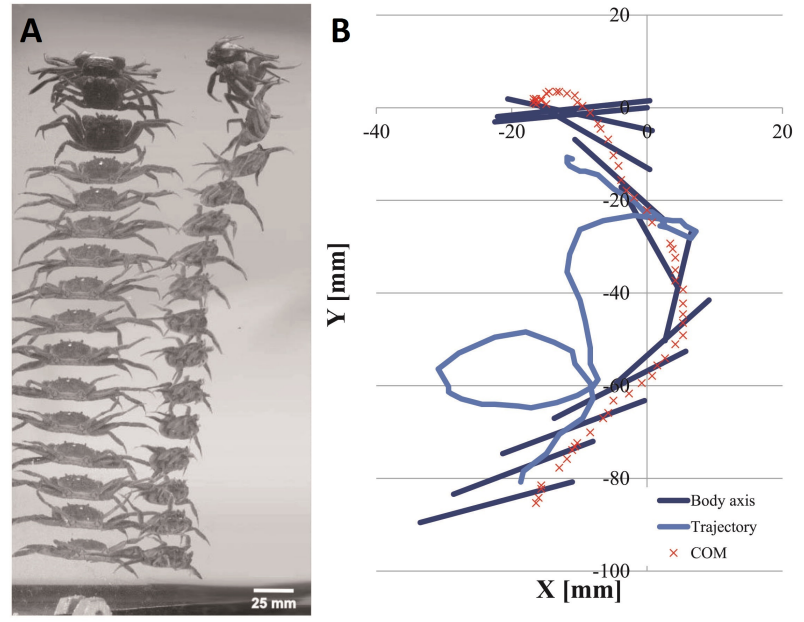

FiguRE 2. A. Stereotypical turning maneuvers observed for $E$. sinensis after a virtually moment-free start (9, modified). $\Delta \mathrm{t}=0.2 \mathrm{~s}$. B. Positions of body axis, right 5 th leg and COM for one individual (E. s.). Origin of ordinates on caudal end of carapace at starting point. $\Delta \mathrm{t}=0.02 \mathrm{~s}(0.1 \mathrm{~s}$ for body axis $)$.

\subsubsection{EXPERIMENTS}

The performed tests are adapted by the setup of the experiments carried out in section 2.1.1. Therefore, the robot starts in a supine position at the top of the tank. To initialize the turning, the two legs will strike simultaneously. The appropriate kinematic parameters were determined by an iteratively change of the striking angle $\theta$ at the maximum angular striking velocity $\omega$ of $4.76 \mathrm{~s}^{-1}$. The acting forces were kinematically analyzed by equation 1 .

\section{Results}

Falling E. sinensis show a body rotation along the transversal axis during free fall (see Figure 2A). The rotation is initiated directly after release by one stroke cycle of the 5th leg pair followed by a passive phase and stopped again with the 5 th leg pair by a counterstrike. The turn is completed in $0.76 \pm 0.15 \mathrm{~s}$ which leads to a mean angular velocity of $\omega=4.3 \mathrm{~s}^{-1}$. In Figure $2 \mathrm{~B}$ the trace of one individual turning maneuver is given in sagittal view. Besides the reorientation of the body axis the trajectory of the 5th leg tip is given to show the efficient strike and counterstrike. The moving location of the COM testifies the complex interaction of the acting forces.

\subsection{Force Calculation of 5TH Leg Pair BY KINEMATIC APPROACH AND PIV}

Forces which are induced by moving the hind leg pair in phase 2a (see Figure 3B) have a maximum at 33\% of the stroke with a mean force of $0.018 \pm 0.004 \mathrm{~N}$ and a maximum peak of $0.023 \pm 0.002 \mathrm{~N}$. The total momentum during phase $2 \mathrm{a}$ is 0.0036 Ns. For phase $3 \mathrm{a}$ the maximum is at $35 \%$ of leg stroke with a mean force $0.015 \pm 0.003 \mathrm{~N}$ and a maximum peak of 0.018 $\pm 0.002 \mathrm{~N}$.

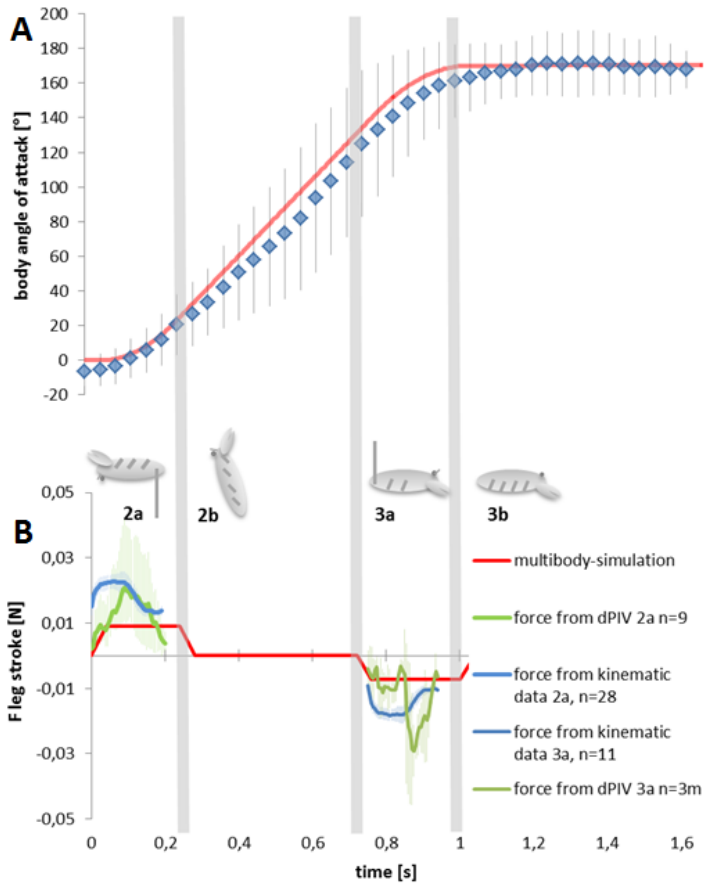

Figure 3. Results from experiment and multibodysimulation for A: body angle and B: induced forces. Experimental forces are mean values of the force calculations. Body angle of experimental data (blue rhomb), $\mathrm{n}=8$ and multibody-simulation (red line) are showing the same trend in time and angle. The calculated forces are 1.5 to 2 times higher (PIV: $2 \mathrm{a} \mathrm{n}=9,3 \mathrm{a} \mathrm{n}=3$, Kinematic: $2 \mathrm{a} n=28,3 \mathrm{a} n=11$ ) than the forces used in the simulation.

The PIV data shows that propulsive forces were produced by the hind legs to initiate and stop the rotation. Peak forces to initiate the rotation $(45 \%$ of stroke cycle) of $0.021 \mathrm{~N}( \pm 0.001 \mathrm{~N})(\mathrm{n}=9)$ lead to a total momentum of 0.0021 Ns.

\subsection{Simulation}

The simulation shows that a constant force of $0.0045 \mathrm{~N}$ acting on each hind leg respectively for $0.2 \mathrm{~s}$ leads to a successful rotation within time. This means that the total momentum of $0.0018 \mathrm{Ns}$ causes a constant angular velocity of $3.99 \mathrm{~s}^{-1}$ in the middle part of the turning maneuver, part $2 \mathrm{~b}$ in Figure 3 .

\subsection{Technical Model}

The experiments confirmed the assumption, that a stroke with two legs is sufficient for a $180^{\circ}$-turn (see Figure $4 \mathrm{~B}$ ). The correspondent kinematic parameters are $\theta=2.44$ and $\omega=4.76 \mathrm{~s}^{-1}$. The complete turn was herewith possible within $1.00 \pm 0.12 \mathrm{~s}(\mathrm{n}=6)$. An exemplary run is shown in Figure $4 \mathrm{~B}$. The turn is divisible into the same five phases like E. sinensis. During the initializing (1) the chassis is descending in a supine position( till I/ 2). The striking phase (2a) includes the active leg movement and thereby the acceleration (I-II/ 2-3). The following gliding phase (2b) describes the passive turning based on inertia (II-III/ 


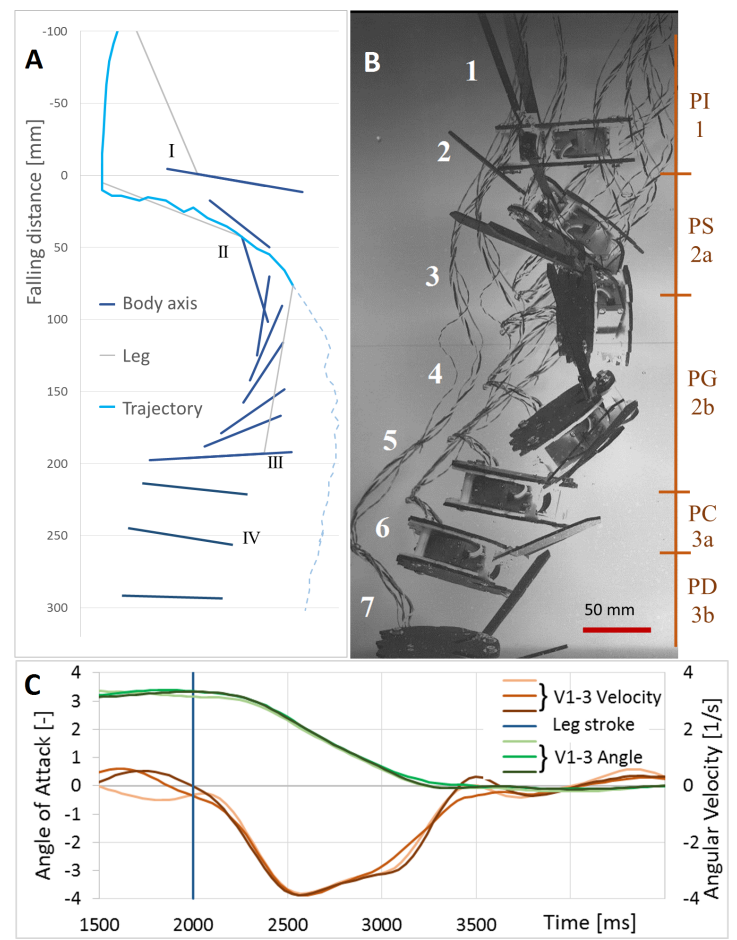

Figure 4. Turning movement of O-Typus. B. observed turning behavior with five characteristic Phases (Initializing PI스, Striking PS스르, Gliding $\mathrm{PG} \hat{=} 2 \mathrm{~b}$, Counterstriking $\mathrm{PC} \hat{=} 3 \mathrm{a}$, Descending $\mathrm{PD} \hat{=} 3 \mathrm{~b})$; $\varnothing \Delta \mathrm{t}=0,36 \mathrm{~s}$. A. Course of the body axis and trajectory of the left leg's tip. Leg positions at the end of PI (I), end of PS (II) and the target position (III) in grey. IV shows the maximal overwinding; $\Delta \mathrm{t}=0,2 \mathrm{~s}(0,1 \mathrm{~s}$ during passive turning (II-III)). C. Angle of attack (green) and angular velocity (red) of three runs.

3-5). When the horizontal target position is reached, the counterstriking phase (3a) starts with a counter movement of the legs, to stop the rotation (III-IV/ 5-6). Thereafter the chassis descends (3b) in a stable up-right position.

Additionally Figure 4 A shows the trajectory of one striking leg. Point I poses the initiation of the stroke, Point II the end of the stroke, Point III the end of the $180^{\circ}$-turn and thereby the initiation of the counterstrike and Point IV the eventual slight overwinding.

The graphs of Figure $4 \mathrm{C}$ are describing the shifting of the angle of attack and its angular velocity. The same phases can be located here. The stroke starts at $2000 \mathrm{~ms}$ and leads over from phase 1 to $2 \mathrm{a}$. The maximal angular velocity of $4.06 \mathrm{~s}^{-1}$ is reached at the end of 2a. Subsequently $\omega$ starts decreasing within phase 2b. After approximately $1150 \mathrm{~ms}$ the turn is completed and results in phase $3 \mathrm{a}$, which prevents overwinding. Afterwards the body descends stable with minor wobbling.

The force which is induced by the moving leg pair in phase $2 \mathrm{a}$ has a mean value of $0.0175 \mathrm{~N}$. This propulsive force is acting over $0.51 \mathrm{~s}$, which results in a total generated momentum of $0.00898 \mathrm{Ns}$.

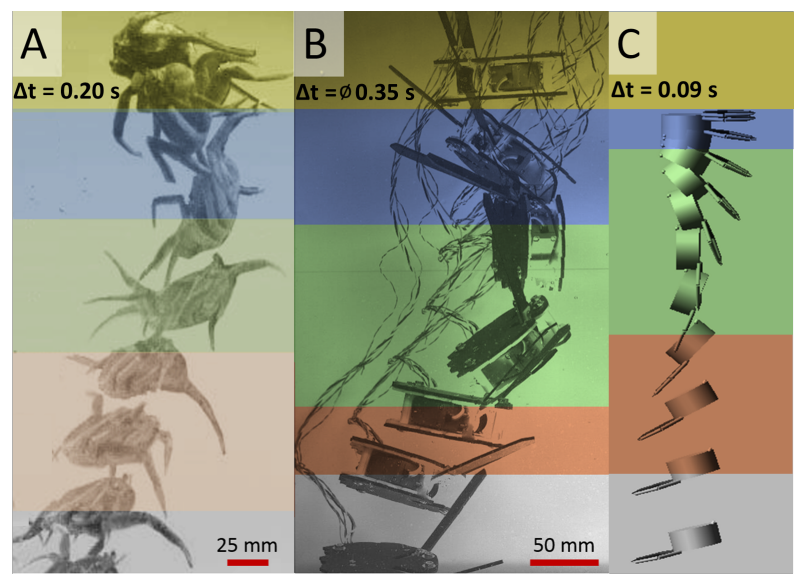

FiguRE 5. Comparison of characteristic turning phases of (A) E. sinensis, (B) O-Typus and (C) the simulated model. Phases: 1 (yellow), 2a (blue), 2b (green), 3a (orange) and 3b (grey).

\section{Conclusions}

\subsection{COMPARISON OF E. sinensis With ITS MODELS}

A qualitative comparison of the righting behavior of E. sinensis with the turning characteristics of the adapted robot and the multibody simulation is shown in Figure 5 O-Typus shows like the biological model a complete rotation after a single stroke. Its rotation could be divided into the exact same phases like E. sinensis. The correspondence can be seen by comparing the phases. The duration of some phases are partially varying. A considerable difference between $\mathrm{A}$ and $\mathrm{B}$ can be seen within phase 2b. According to this, the larger O-Typus needs significantly more time to complete the passive turn.

A full comparison of the designed and measured parameters is shown in Table 1. While the numerical model could be simulated in the original scale, the robot had to be enlarged to fit all devices. However the mean density was adapted. The measured propulsive forces of E. sinensis deviate among the two methods. While the PIV data corresponds closely to the values gained by the simulation, the kinematic approach matches nicely with the acting forces on O-Typus. Due to the considerable bigger dimensions of O-Typus, the total momentum to generate a similar turn is about 3 times of E. sinensis value. Herewith O-Typus reaches a mean angular velocity of $3.93 \pm 0.30 \mathrm{~s}^{-1}$, which deviates no more than $8.6 \%$ of its model.

\subsection{Transferability to Aquatic Robots}

As the motivation for this study showed, there is a desperate need in new stabilizing systems for technical aquatic applications. The observed and tested righting behavior of $E$. sinensis revealed a high efficiency and therefor a potential approach to solve the problem. In order to verify, whether this new stabilizing system is an optimization compared to current methods, further 


\begin{tabular}{|c|c|c|c|c|c|}
\hline \multirow[t]{2}{*}{ Parameter } & & \multicolumn{2}{|c|}{ E. sinensis } & \multirow[t]{2}{*}{ Simulation } & \multirow[t]{2}{*}{ Robot } \\
\hline & & Kinematic & PIV & & \\
\hline Scaling & & \multicolumn{2}{|c|}{$100 \%$} & $100 \%$ & $200 \%$ \\
\hline Mass & {$[\mathrm{g}]$} & \multicolumn{2}{|c|}{88.65} & 87 & 220 \\
\hline Mean Density & {$\left[\mathrm{g} / \mathrm{cm}^{3}\right]$} & \multicolumn{2}{|c|}{1.12} & 1.12 & 1.15 \\
\hline Mean Force & {$[\mathbf{N}]$} & $\begin{array}{c}0.018 \\
\pm 0.004\end{array}$ & $\begin{array}{c}0.011 \\
\pm 0.003\end{array}$ & 0.009 & 0.0175 \\
\hline Momentum & {$[\mathrm{Ns}]$} & 0.0036 & 0.0021 & 0.0018 & 0.00898 \\
\hline Mean Angular & 1. $\left[\mathrm{s}^{-1}\right]$ & \multicolumn{2}{|c|}{4.30} & 3.99 & $3.93 \pm 0.30$ \\
\hline
\end{tabular}

TABLE 1. Summary of relevant parameters regarding the turning of E. sinensis, its numerical model and its technical application. Values of the measured force are mean values during the striking phase 2a. The angular velocity is the averaged value of the whole turn.

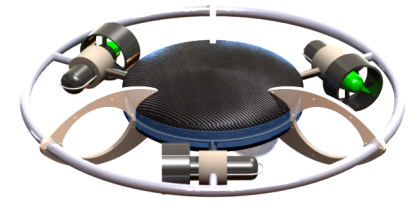

FiguRE 6. Smart-E. Modern thruster-driven AUV, which is declared to be highly efficient [10].

studies have to be carried out. Thus, two different efficiencies have to be compared.

On one hand, the power consumption has to be evaluated. At the current state of research no statement is possible, as there is no data about robotic devices, driven by thruster, available to compare it with. It would be worth to use an existing modern Robot like the AUV Smart-E [10, to compare the two mechanisms at the same chassis.

On the other hand, time efficiency has to be considered. Most remotely operated vehicles are featuring slow turning capabilities. Unfortunately non published the specific turning kinematics. However, the maximum angular velocity of $4.25 \mathrm{~s}^{-1}$ reached by $\mathrm{O}$ Typus is considerably (visually) higher than of most found thruster-driven devices.

\section{ACKNOWLEDGEMENTS}

Special thanks are referring to the Department of Biomimetics in Bremen, which made these studies possible.

\section{REFERENCES}

[1] G. Krummel, K. N. Kaipa, S. K. Gupta. A horseshoe crab inspired surf zone robot with righting capabilities. In Proc. of the ASME 2014 Int. Design Eng. Tech. Conf. \& Comput. and Inform. in Eng. Conf. 2014. DOI:10.1115/DETC2014-34679
[2] E. Chang-Siu, T. Libby, M. Tomizuka, R. J. Full. A Lizard-inspired active Tail enables rapid Maneuvers and dynamic Stabilization in a terrestrial Robot. IEEE/RSJ Int Conf of Intelligent Robots and Syst 2011. DOI:10.1109/IROS.2011.6094658

[3] P. Bausch, F. Hoffmann, A. B. Kesel. Im freien Fall unter Wasser - Lagestabilisierung bei Wollhandkrabben. Eine experimentelle Analyse. In Bionik: Patente aus der Natur (ed. A.B. Kesel und D. Zehren). 155-161, Bionik-Innovations-Centrum, Bremen, 2015.

[4] F. Hoffmann, S. Labisch, R. Sonntag, et al. Analysis of underwater Righting Reflex of the Chinese mitten crab (Eriocheir sinensis) using PIV and Numerical Multibody Simulation. In Proceedings of the Annual Main Meeting of the Society for Experimental Biology, Prag, [A 11.68]. 2015.

[5] T. Riphaus, S. Labisch. Konstruktion des biologisch inspirierten Roboters O-Typus zur Implementierung und Untersuchung des Stabilisationsverhaltens von Decapoden. In Proceedings of the 7. IEEE Germany Student Conference, Magdeburg. 2016.

[6] W. Thielicke, E. J. Stamhuis. PIVlab - towards Userfriendly, affordable and accurate Digital Particle Image Velocimetry in MATLAB., 2014. J.OpenRes.Softw . , 2, . DOI:http://doi.org/10.5334/jors.bl

[7] J. M. V. Rayner. A new Approach to Animal Flight Mechanics. J Exp Biol 80:17-54, 1979.

[8] G. R. Spedding, J. M. V. Rayner, C. J. Pennycuick. Momentum and Energy in the Wake of a Pigeon (Columba livia) in Slow Flight. J Exp Biol 111:81-102, 1984.

[9] P. Bausch, P. Berger, F. Hoffmann, A. B. Kesel. How to land on your Legs - Underwater Righting Reflexes in Crabs and Crayfish. In Proceedings of the Annual Main Meeting of the Society for Experimental Biology, Prag, [A 11.67]. 2015.

[10] B. Meyer, K. Ehler, C. Osterloh, E. Maehle. An autonomous omnidirectional Underwater Robot. Journal of Behavioral Robotics pp. 204-210, 2013. 